\title{
Some Thoughts on Behavioral Programming
}

\author{
David Harel \\ Weizmann Institute of Science, Israel \\ dharel@weizmann.ac.il
}

\begin{abstract}
The talk starts from a dream/vision paper I published in 2008, whose title, Can Programming be Liberated, Period?, is a play on that of John Backus' famous Turing Award Lecture (and paper). I will propose that or rather ask whether programming can be made a lot closer to the way we humans think about dynamics, and the way we somehow manage to get others (e.g., our children, our employees, etc.) to do what we have in mind. Technically, the question is whether we can liberate programming from its three main straightjackets: (1) having to directly produce a precise artifact in some language; (2) having actually to produce two separate artifacts (the program and the requirements) and having then to pit one against the other; (3) having to program each piece/part/object of the system separately. The talk will then get a little more technical, providing some evidence of feasibility of the dream, via LSCs and the play-in/play-out approach to scenario-based programming, and its more recent Java variant. The entire body of work around these ideas can be framed as a paradigm, which we call behavioral programming.
\end{abstract}

